\title{
Analyse expérimentale des phénomènes d'interaction d'une machine et de son circuit
}

\author{
D. Garreau, \\ B. Leducq \\ EDF-DER, Chatou \\ J.L. Trolle \\ Société Bertin, Plaisir \\ EDF-DER, Saint-Denis
}

L'interaction d'une machine avec son circuit constitue un problème industriel important. En effet, ce phénomène peut engendrer des vibrations excessives des composants du circuit: machine elle-même, tuyauteries, vannes, supportages. Nous présentons dans cet article les études expérimentales en cours dans nos différents laboratoires.

En premier lieu, les moyens expérimentaux utilisés pour cette étude (boucle d'essais) sont décrits.

Ensuite, les différentes méthodes d'analyse expérimentale sont présentées. L'accent est mis sur la validation de modèles (ondes planes, intensimétrie hydroacoustique, matrice interspectrale) ainsi que sur la localisation et la caractérisation des sources acoustiques.

Finalement, des résultats sont présentés; ils portent sur des études de laboratoire et sur des cas semi-industriels. Les problèmes rencontrés lors de l'analyse de ces cas sont soulignés.

\section{Introduction}

L'endommagement des circuits hydrauliques et de certains composants importants (pompe, vanne,...) a parfois pour origine des phénomènes dynamiques (vibrations, fluctuations de pression). Par ailleurs, de part la nature propagative de ces phénomènes, il n'est pas rare de constater des dégradations sur des composants du circuit éloignés de la source d'excitation, voire sur des circuits annexes. Le premier problème, auquel l'expérimentateur se trouve confronté, consiste bien souvent à localiser les sources prépondérantes. De plus, le caractère acoustique des mécanismes associés à ces sources fait parfois apparaitre des interactions, que ce soit entre les différents éléments du circuit ou entre l'écoulement et les vibrations de tuyauteries. Actuellement, les moyens d'étude et d'analyse expérimentale sont généralement limités à des informations "statiques " (débit, NPSH, valeur efficace de la fluctuation de pression,...) ou à des données spectrales brutes (densité spectrale, fonction de transfert,...).

En règle générale, ces informations sont insuffisantes à l'établissement d'un diagnostic, et a fortiori à la définition des remèdes à mettre en œuvre. En effet, ces informations n'intègrent pas le comportement dynamique des phénomènes physiques mis en jeu, ce qui explique leurs carences.

A notre avis, le diagnostic doit s'appuyer sur une démarche expérimentale incluant, lorsque cela est possible, l'étude des points suivants :

- sources d'excitations (localisation, niveaux relatifs)

- comportement modal du circuit (résonances hydrauliques et mécaniques)

- réponse dynamique en fonctionnement.

Experimental analysis of the interaction phenomena of a pump and its circuit.

The interaction of a machine with its circuit may generate excessive vibrations of the components. Current studies show the experimental means and the analysis methods of the results of the experiments. The emphasis is placed on the validation of models and on the localization and characterization of acoustic sources. The results submitted are then related to laboratory tests and semi-industrial cases. The problems encounted when analysing these cases are emphasized. 


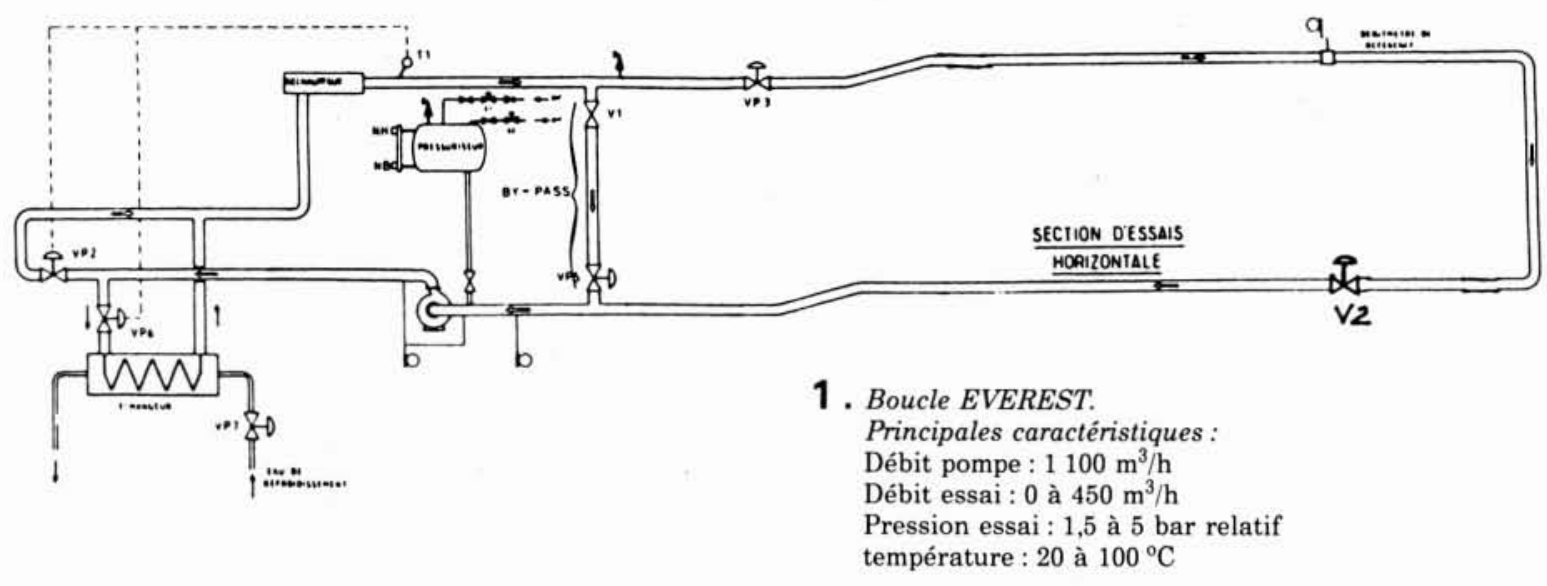

\section{Moyens expérimentaux et descriptif des essais}

\subsection{Présentation de la boucle Everest $(E D F / D E R)$}

La boucle Everest (figure 1) comporte un tronçon rectiligne d'essai de diamètre $100 \mathrm{~mm}$ et d'une longueur de $15 \mathrm{~m}$ environ. Le débit dans l'épingle d'essai et le débit dans la pompe sont réglés de façon indépendante par l'intermédiaire d'un by-pass. Les caractéristiques de la pompe et du circuit permettent d'obtenir un débit maximum dans l'épingle de l'ordre de $450 \mathrm{~m}^{3} / \mathrm{h}$ et une perte de charge de 5 bars.

\subsection{Descriptif des essais}

Les fluctuations de pression ont été mesurées en amont et en aval de la vanne test V2 afin de déterminer, à partir d'un traitement intensimétrique, la part des fluctuations de pression de nature propagative générées par cette vanne papillon (figure 2). Par ailleurs, des mesures de fluctuations de pression sont réalisées à l'aval immédiat de la vanne afin d'estimer l'évolution spatiale des fluctuations locales de pression; ces mesures font l'objet d'un traitement particulier.

Les résultats présentés dans cet article concernent l'étude par une méthode d'intensimétrie hydro-acoustique de la vanne en régime cavitant, ainsi que sur l'utilisation des matrices interspectrales pour la détection des sources acoustiques en régime non cavitant.

\section{Méthode d'analyse}

\subsection{Intensimétrie hydro-acoustique}

La méthode d'intensimétrie hydro-acoustique utilisée est basée sur un modèle de propagation des fluctuations de pression en ondes longitudinales planes. Les équations de propagation sont alors monodimensionnelles. La fluctuation de pression s'exprime sous la forme:

$$
P(x, f)=A(f) e^{-i k x}+B(f) e^{i k x}
$$

où $k=\frac{2 \pi f}{c}$ en première approximation.

L'expression de la fluctuation de débit est analogue :

$$
Q(x, f)=\frac{S}{\rho c}\left[A(f) e^{-i k x}-B(f) e^{i k x}\right]
$$

La puissance acoustique propagée est donnée par la relation :

$$
\text { P.A. }(f)=\operatorname{Re}\left[P(x, f) Q^{*}(x, f)\right]
$$

De plus, par convention, on appelle :

- puissance acoustique directe (P.A.D.), la puissance acoustique de l'onde directe $A(f)$, c'est-à-dire celle qui se propage dans le sens de l'écoulement pris comme référence,

- puissance acoustique inverse (P.A.I.), la puissance acoustique de l'onde inverse $B(f)$, c'est-à-dire celle qui se propage dans le sens contraire de l'écoulement,

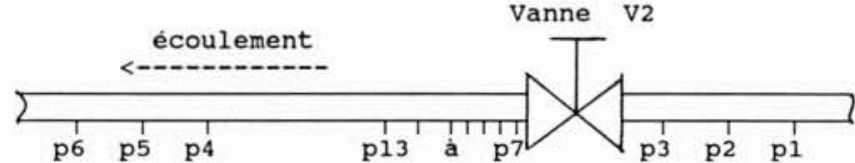

2. Implantation des capteurs de pression fluctuante.

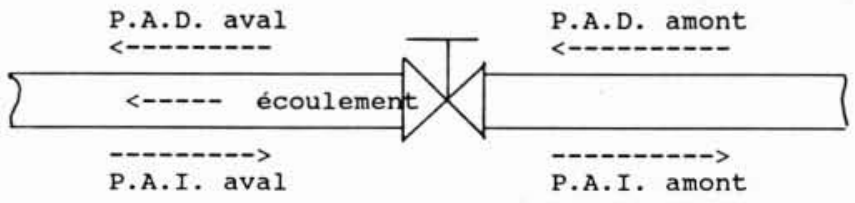

3. Schéma conventionnel. 
- puissance acoustique globale (P.A.G.), la puissance acoustique totale, somme des puissances directe et inverse.

Ces puissances acoustiques sont représentées sur la figure 3 et s'expriment sous la forme :

$$
\begin{gathered}
\text { P.A.D. }=\frac{S}{\rho c}|A(f)|^{2} \\
\text { P.A.I. }=\frac{S}{\rho c}|B(f)|^{2} \\
\text { P.A.G. }=\frac{S}{\rho c}\left[|A(f)|^{2}-|B(f)|^{2}\right]
\end{gathered}
$$

Ces équations de puissances acoustiques permettent de déterminer l'amplitude des ondes directe et inverse.

\subsection{Intérêt d'un traitement intensimétrique}

\subsubsection{Indépendance vis-à-vis de la position}

La fluctuation de pression, qui est la grandeur physique immédiatement accessible à la mesure, présente l'inconvénient d'être dépendante de la position du capteur sur la ligne de tuyauterie. Cet aspect ne permet pas la comparaison directe de deux mesures de fluctuation de pression. A titre d'exemple, le tableau ci-dessous résume l'évolution de l'amplitude de la raie correspondant à la fréquence de passage des aubes d'une pompe à l'aspiration et au refoulement de celle-ci.

\begin{tabular}{|l|c|c|c|c|c|c|c|c|c|c|}
\cline { 2 - 9 } \multicolumn{1}{c|}{} & \multicolumn{9}{c|}{ Aspiration } & \multicolumn{3}{|c|}{ Refoulement } \\
\hline Point de mesure & 1 & 2 & 3 & 4 & 5 & 6 & 7 & 8 & 9 & 10 \\
\hline $\begin{array}{l}\text { Amplitude } \\
\text { du pic } \\
\text { imbart }\end{array}$ & 20 & 33 & 40 & 38 & 12 & 16 & 20 & 28 & 25 & 22 \\
\hline
\end{tabular}

Cette évolution montre qu'il est délicat d'estimer le niveau des fluctuations de pression en dehors des points de mesure. L'analyse réalisée à l'aide des puissances acoustiques a montré que l'amplitude maximale est de 47 mbar à l'aspiration et de 28 mbar au refoulement.

\subsubsection{Indépendance vis-à-vis de la vitesse du son}

La célérité du son constitue un autre élément à prendre en compte dans l'analyse des fluctuations de pression. Les variations de ce paramètre rendent délicate toute comparaison réalisée sur un même point de mesure pour différents essais, en particulier si le taux de vide dans le circuit a varié.

L'examen de l'équation de propagation (1) des fluctuations de pression témoigne de la quasi-impossibilité de corriger les résultats de cette influence. Il est donc indispensable de connaître les ondes acoustiques directe et inverse $A(f)$ et $\mathrm{B}(f)$ dans le circuit, qui sont, elles, indépendantes de la célérité du son. Les puissances acoustiques, qui présentent l'inconvénient d'y être sensible, peuvent par contre en être corrigées (4), (5), (6).

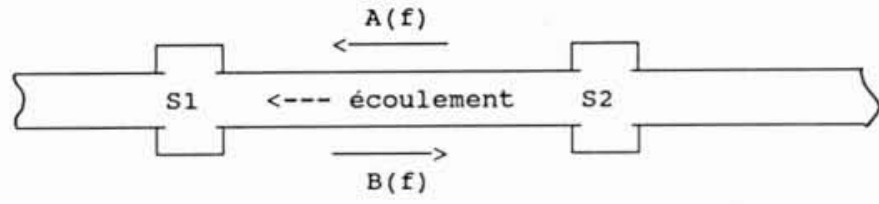

4. Configuration élémentaire du circuit.

\subsubsection{Localisation de la source prépondérante}

Sur la figure 4 sont représentées les ondes directe et inverse entre deux sources acoustiques (pompe, vanne,...). Cette configuration est définie comme la structure élémentaire d'un circuit, celui-ci étant un assemblage de ces éléments.

Si la source acoustique $S_{1}$ est prépondérante, l'amplitude de l'onde directe $A(f)$ sera supérieure à celle de $B(f)$. Dans ce cas, la puissance acoustique globale sera positive, indiquant ainsi que la principale source acoustique est $S_{1}$ :

$$
\text { P.A.G. }=\frac{S}{\rho c}\left[|A|^{2}(f)-|B|^{2}(f)\right]>0
$$

Le bilan des puissances directe et inverse peut s'avérer insuffisant pour déterminer les niveaux relatifs de chacune des sources dans le cas où celles-ci sont du même ordre de grandeur ou que le coefficient de réflexion de l'une est nettement supérieur à celui de l'autre.

Il faut alors avoir recours à une analyse plus complexe qui nécessite l'examen des phases des ondes propagées de manière à estimer les retards, donc les distances entre les sources et les positions de réflexion des ondes acoustiques.

\section{Analyse par intensimétrie}

L'essai a porté sur une vanne papillon lors d'un régime cavitant proche du débit de blocage.

\subsection{Analyse des autospectres de pression (figure 5, page suiv.)}

Les autospectres de pression à l'amont et à l'aval ont été déterminés dans la bande de fréquences 0-260 Hertz.

Ces spectres présentent une succession de raies; ces raies ne sont pas caractéristiques de la rotation de la pompe. Par ailleurs, les fréquences des raies sont très différentes de part et d'autre de la vanne. Leurs amplitudes varient selon le point de mesure, ce qui laisse présager la nature acoustique des fluctuations de pression.

Par ailleurs, on constate à l'aval de la vanne l'existence de fluctuations de pression large bande vers les plus hautes fréquences; celles-ci sont caractéristiques de la cavitation. 
PRAM $\gg$ MODULE INTENSIMETRIE - EDF/DER/EP/REME/EM ESSAI VANNE PAPILLON EN REGIME CAVITANT PAGE

Autospectres amont - capteurs 1, 2, 3

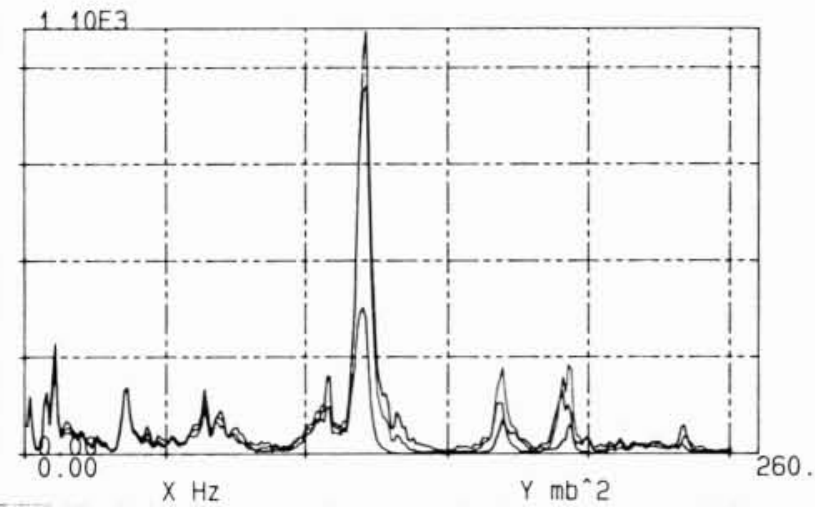

Autospectres aval - capteurs 4,5,6

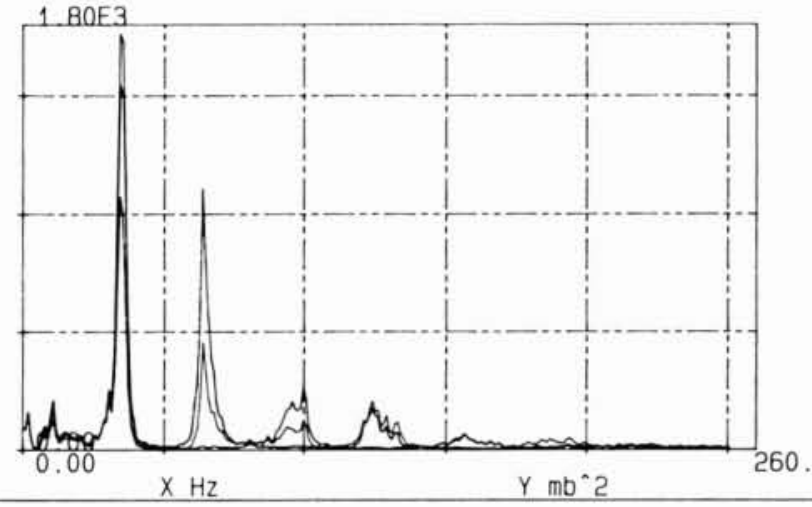

5.

PRAM $\gg$ MODULE INTENSIMETRIE - EDF/DER/EP/REME/EM ESSAI VANNE PAPILLON EN REGIME CAVITANT PAGE 1

Fluctuation locale de pression $3-35 \mathrm{~Hz}$

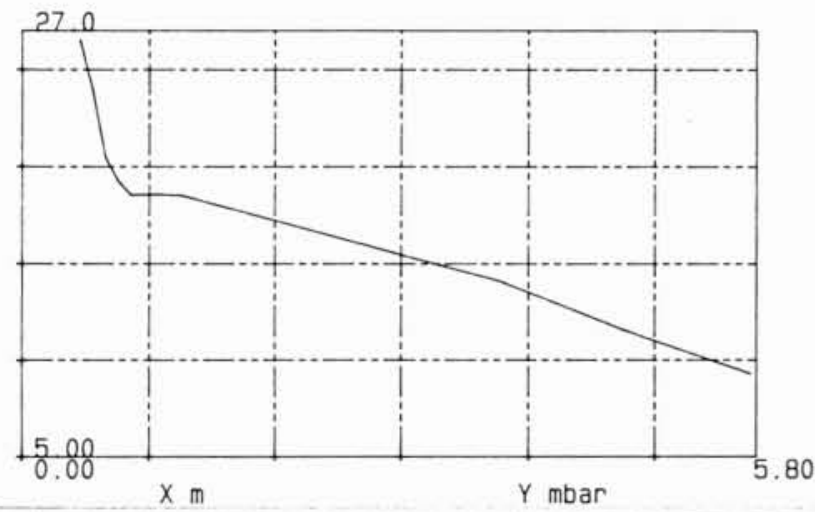

Fluctuation locale de pression $35-230 \mathrm{~Hz}$

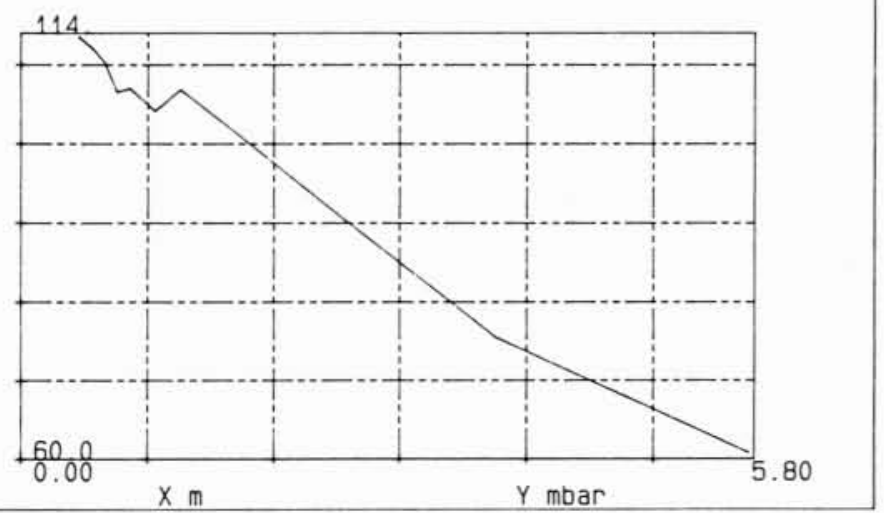

PRAM $\gg$ MODULE INTENSIMETRIE - EDF/DER/EP/REME/EM ESSAI VANNE PAPILLON EN REGIME CAVITANT PAGE 1

Vitesse du son mesuree amont

Vitesse du son mesuree aval
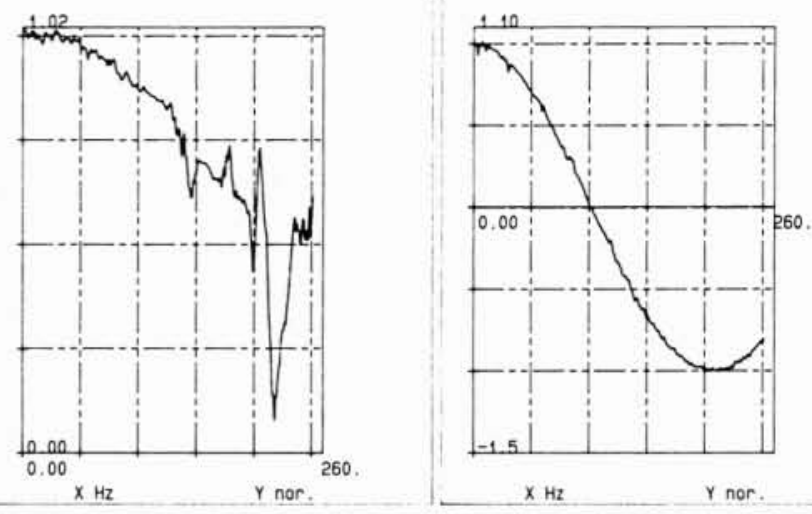

Puissance acoustique globale amont

Puissance acoustique globale aval
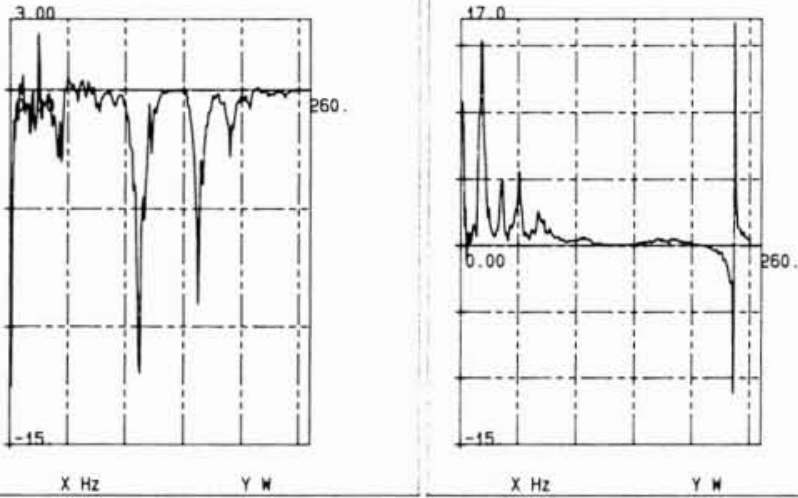

6.

PRAM $\gg$ MODULE INTENSIMETRIE - EDF/DER/EP/REME/EM ESSAI VANNE PAPILLON EN REGIME CAVITANT PAGE 1

Rapport onde inverse/onde directe (module)

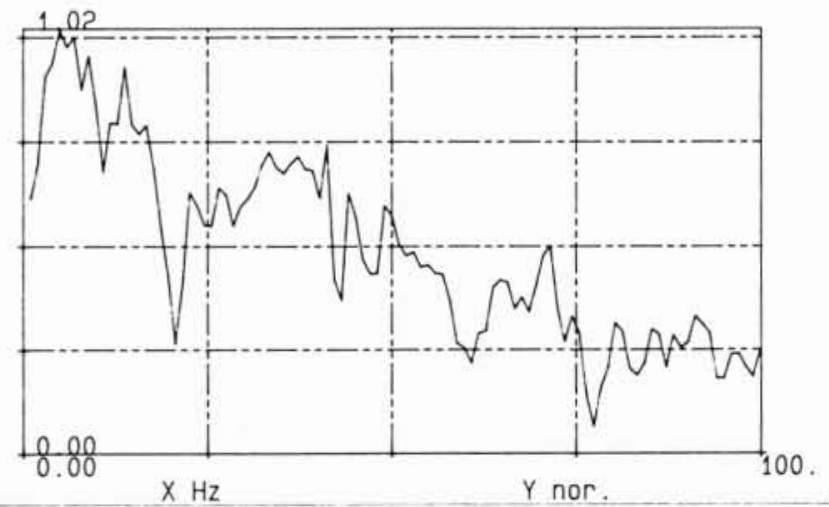

Rapport onde inverse/onde directe (phase)

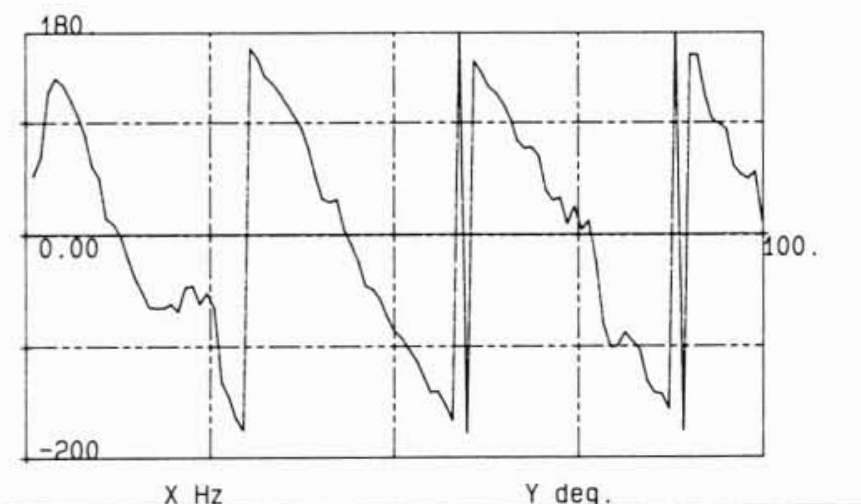




\subsection{Analyse par intensimétrie acoustique}

\subsubsection{Détermination de la célérité du son (figure 6)}

La vitesse du son est estimée à partir de la demi-somme des fonctions de transfert entre les capteurs extrêmes et le capteur médian. Si les capteurs sont équidistants, la demi-somme des fonctions de transfert a une allure de fonction cosinus [1] :

$$
\begin{aligned}
R=(P(x 3, f)+P(x 1, f)) / 2 & \text { * } P(x 2, f) \\
& =\cos \left(2 \pi f^{*}(x 3-x 2) / C\right)
\end{aligned}
$$

A l'amont, comme à l'aval, la courbe est caractéristique de la propagation sous la forme d'ondes planes, et ceci même en présence de forte cavitation.

Pour cet essai, la célérité est de $1260 \mathrm{~m} / \mathrm{s}$ à l'amont et de $473 \mathrm{~m} / \mathrm{s}$ à l'aval au niveau des capteurs 4.5.6. A l'aval, la célérité varie avec l'éloignement de la vanne : $380 \mathrm{~m} / \mathrm{s}$ à $50 \mathrm{~cm}, 420 \mathrm{~m} / \mathrm{s}$ à $85 \mathrm{~cm}$ et $473 \mathrm{~m} / \mathrm{s}$ à $4,75 \mathrm{~m}$.

On assiste probablement à une légère résorption des bulles de cavitation.

\subsubsection{Puissances acoustiques (figure 6)}

Les puissances acoustiques globales présentent des spectres de raies négatives à l'amont et positives à l'aval. Ceci signifie que l'émission a lieu dans le sens inverse de l'écoulement à l'amont de la vanne et dans le sens de l'écoulement à l'aval; par conséquent, la source prépondérante est la vanne.

Seule la raie à $25 \mathrm{Hertz}$, qui correspond à la rotation de la pompe, a une puissance globale positive à l'amont et à l'aval. A ce régime de fonctionnement, cette partie du circuit est essentiellement sensible à l'émission d'ondes acoustiques engendrées au refoulement de la pompe.

Hormis la raie à 25 Hertz, aucune autre raie n'est présente à la fois en amont et en aval de la vanne. La vanne, qui joue le rôle de source excitatrice, conditionne ces raies qui correspondent probablement aux résonances hydrauliques des circuits amont et aval. A l'aval, la puissance globale présente un saut vers 230 Hertz qui correspond à la première fréquence d'indétermination de la méthode. Pour lever cette indétermination, il faudrait disposer de mesures supplémentaires, obtenues avec des capteurs distants d'un autre intervalle.

\subsubsection{Fluctuations locales de pression (figure 7)}

Le traitement par intensimétrie permet de déterminer les fluctuations de pression en ondes longitudinales planes qui sont les seules susceptibles de se propager dans l'ensemble du circuit pour les fréquences considérées (inférieures à quelques kiloHertz). Par conséquent, si l'on retranche à la fluctuation de pression mesurée à l'aval immédiat de la vanne, la fluctuation à caractère propagatif mesurée en champ lointain après l'avoir transportée en cette position, on obtient la fluctuation locale de pression.

Les spectres de fluctuation locale de pression contiennent une partie basse fréquence, typiquement inférieure à $50 \mathrm{Hertz}$, ainsi qu'une partie plus haute fréquence relative à la cavitation. En effet, la cavitation émet dans toutes les directions, mais seulement une faible partie se propage sous la forme d'ondes planes dans l'axe de la tuyauterie, le reste n'ayant qu'une influence locale. L'évolution des fluctuations locales de pression est représentée en fonction de la distance entre la mesure et la vanne. Le spectre basse fréquence présente une décroissance plus brutale au voisinage de la vanne, tandis que le spectre haute fréquence évolue de façon quasi-linéaire. Il est ainsi possible de définir des longueurs de corrélation de l'aspect local de ces phénomènes.

\section{Comportement modal hydraulique du circuit (figure 8)}

Lors des essais en régime cavitant, des raies, qui ne sont pas caractéristiques de la pompe, sont apparues sur les spectres en amont et en aval de la vanne. Celles-ci sont dues à des phénomènes de résonance hydraulique liés à l'interaction entre l'émission de la vanne et les réflexions dans le circuit. Afin de déterminer les conditions de résonance, il est nécessaire de localiser la position de réflexion des ondes acoustiques. la figure 9 représente une configuration élémentaire de circuit réflecteur. L'expression de l'onde inverse $B(f)$ s'obtient en fonction de l'onde directe $A(f)$ et du coefficient de réflexion par la relation :

$$
\mathrm{B}(f)=A(f) \operatorname{Re}^{i \theta} e^{\frac{-4 i \pi / L}{c}}
$$

$\operatorname{Re}^{i \theta}:$ coefficient de réflexion

$e^{\frac{-4 i \pi / L}{c}}:$ déphasage lié au retard de l'onde inverse par

Par conséquent, le module du coefficient de réflexion est égal au module du rapport $B(f) / A(f)$. De plus, la phase de ce rapport évolue linéairement avec la fréquence, permettant ainsi d'obtenir la distance entre la mesure et le lieu de réflexion.

Pour cet essai, la courbe de phase montre que la réflexion de l'onde directe a lieu à 8 mètres environ de la position de mesure, ce qui correspond à l'emplacement d'un divergent dans le circuit. La mesure (point 5) est distante de 5 mètres de la vanne, les fréquences de résonance hydraulique sont obtenues pour une longueur de 13 mètres à partir de l'équation :

$$
F_{R}=k C / 2 l \quad k \in[1,2,3 \ldots]
$$

avec $C=473 \mathrm{~m} / \mathrm{s}, \quad l=13 \mathrm{~m}$

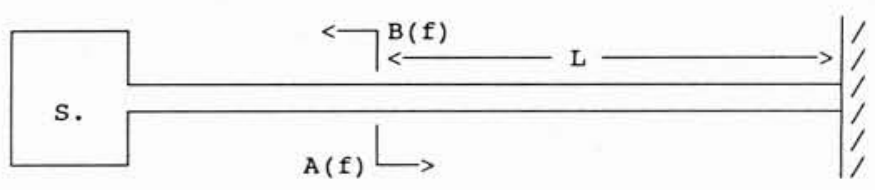

9. Configuration élémentaire du circuit réflecteur. 
Les premières fréquences de résonance sont de 18 Hertz, 36 Hertz,..., à l'aval de la vanne. Ces fréquences sont présentes sur les spectres de puissance acoustique indiquant ainsi l'amplification par le circuit aval de l'émission acoustique de la vanne. Les résonances mises en évidence sont locales et font peu intervenir ce qui se passe en aval du divergent.

\section{Méthode des matrices interspectrales}

\subsection{Présentation de la méthode}

Le principe de la méthode des matrices interspectrales consiste à exploiter les propriétés de la matrice spectrale constituée des interspectres de puissance entre les signaux mesurés. Cette méthode présente un intérêt particulier pour l'étude de la propagation d'ondes planes dans un milieu linéaire. Ces hypothèses physiques sont complétées par des conditions portant sur le caractère stationnaire en espace et en temps des sources, ainsi que sur le caractère homogène et isotrope du milieu.

Le signal mesuré en un point $i$ est considéré comme le produit d'un terme source $a_{j}$ par la réponse impulsionnelle entre le point $i$ et le point $j$ augmenté d'un terme représentant le bruit.

En présence de plusieurs sources décorrélées, la mesure au point $i$ est la somme des signaux en ce même point issus de l'ensemble des sources:

$$
X_{i}(f)=\sum_{k=1}^{N} A_{k}(f) * H_{k i}(f)+B_{i}(f)
$$

La matrice interspectrale est définie par :

$$
\Gamma_{i j}(f)=E\left[X_{i}^{*}(f) * X_{j}(f)\right]
$$

Après développements, en considérant que les sources et les bruits ne sont pas corrélés, la matrice interspectrale se présente sous la forme de deux matrices: l'une représentant les sources, l'autre les bruits.

$$
\begin{aligned}
\Gamma_{i j}(f)=\sum_{k=1}^{N} A_{k}^{*}(f) * A_{k}(f) & * H_{k i}^{*}(f) \\
& * H_{k j}(f)+B_{i}^{*}(f) * B_{j}(f)
\end{aligned}
$$

soit $\Gamma_{i j}(f)=\Gamma(s)_{i j}(f)+\Gamma(b)_{i j}(f)$

On détermine le nombre de valeurs propres significatives de la matrice, ceci donne directement le nombre de sources indépendantes. De plus, les vecteurs propres associés à ces valeurs propres forment une base du sous-espace formé par les sources, ce qui permet ainsi de décomposer la réponse en un point.

\subsection{Application aux essais}

Cette méthode a été utilisée pour l'étude des sources présente lors des essais réalisés sur la vanne papillon en régime non cavitant. L'évolution en fonction de la fréquence des valeurs propres est représentée sur la figure 10. Sur les six valeurs propres déterminées, quatre d'entre elles ont des allures très chaotiques. On constate deux valeurs propres significatives relativement bien séparées : celles-ci correspondent à deux sources distinctes.

Cependant, vers la fréquence $100 \mathrm{Hertz}$, les deux spectres de valeurs propres sont presque confondus ce qui témoigne ainsi d'une dépendance entre ces deux sources.

D'ailleurs, la courbe de détermination de la vitesse du son, obtenue par intensimétrie acoustique réalisée sur cet essai, présente une rupture à cette même fréquence. Cet " accroc " est dû à la déformation dynamique de la tuyauterie : ce phénomène n'est pas pris en compte dans le modèle théorique utilisé dans la méthode d'intensimétrie acoustique. Il y a interaction entre la déformation de la tuyauterie et la fluctuation de pression.

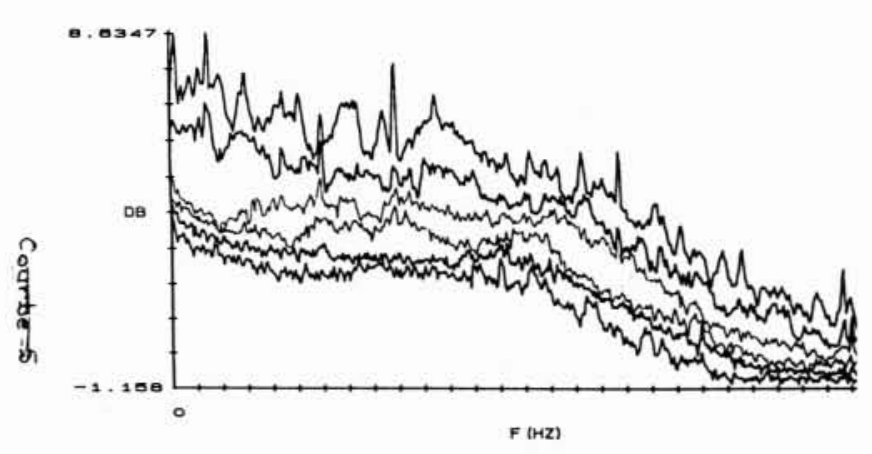

10. Détermination du nombre de sources $S$. Valeurs propres.

\section{Conclusion}

A travers les essais réalisés, les moyens expérimentaux classiques (valeurs efficaces de la fluctuation de pression, données spectrales brutes,...), retenus pour l'analyse des phénomènes dynamiques associés au fluide dans un circuit, ont montré leurs limitations, conséquence directe de l'absence d'intégration de modèle physique dans les résultats. A l'opposé, en ce qui concerne l'analyse des fluctuations de pression dans les tuyauteries, l'intérêt d'une méthode telle que l'intensimétrie acoustique, basée sur un modèle de propagation en ondes planes a été démontré permettant d'obtenir des éléments significatifs de diagnostic. Ainsi, tout en vérifiant la validité d'utilisation d'un tel modèle, les sources acoustiques ont pu être localisées et leur ordre de grandeur estimé. De plus, linteraction entre la vanne et le circuit, sous la forme de résonance hydraulique, a été analysée à partir des résultats obtenus par l'intensimétrie acoustique.

En dernier lieu, la méthode des matrices interspectrales, utilisée pour la caractérisation des sources acoustiques, a montré la dépendance de deux sources, soulignant ainsi une interaction entre la structure et le fluide. Cette méthode semble prometteuse, et son développement devrait être poursuivi. 


\section{Notations}

$\begin{array}{lll}x & : & \text { position le long de la tuyauterie }(\mathrm{m}) \\ L & : & \text { distance avec la position de réflexion }(\mathrm{m}) \\ S & : & \text { section de l'écoulement }\left(\mathrm{m}^{2}\right) \\ f & : & \text { fréquence }(\mathrm{Hz}) \\ f_{R} & : & \text { fréquences de résonance du circuit }(\mathrm{Hz}) \\ \rho & : & \text { masse volumique du fluide }\left(\mathrm{kg} / \mathrm{m}^{3}\right) \\ C & : & \text { vitesse du son dans le fluide }(\mathrm{m} / \mathrm{s}) \\ P(x, f) & : & \text { fluctuation de pression }(\mathrm{Pa}) \\ Q(x, f) & : & \text { fluctuation de débit }\left(\mathrm{m}^{3} / \mathrm{s}\right) \\ A(f) & : & \text { onde acoustique plane directe (dans le sens } \\ & & \text { de l'écoulement) (Pa) } \\ B(f) & : & \text { onde acoustique plane inverse }(\text { dans le sens } \\ & & \text { inverse de l'écoulement) }(\mathrm{Pa}) \\ \text { P.A.G. }(f): & \text { puissance acoustique globale }(W) \\ \text { P.A.D. }(f): & \text { puissance acoustique directe }(W)\end{array}$

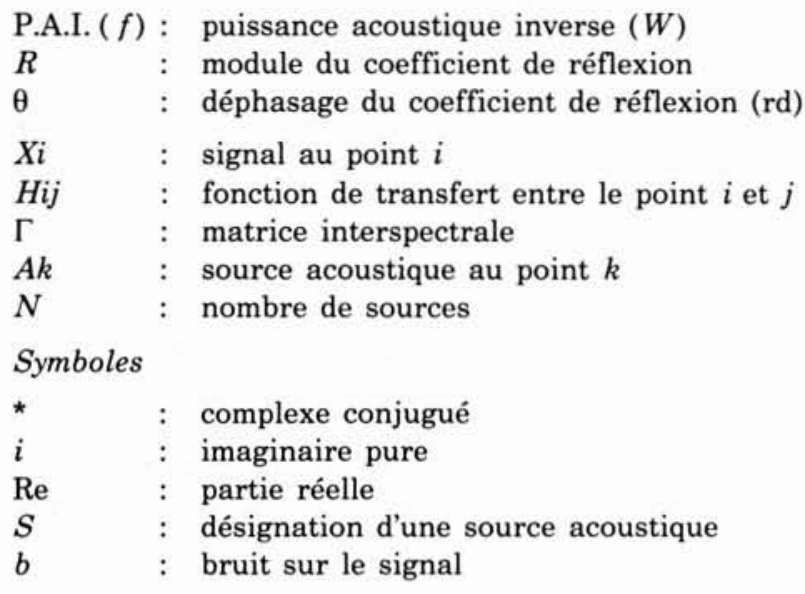

\section{Références}

[1] Margolis, Brown. - " Measurement of the propagation of long-wavelength disturbances through turbulent flow in tubes ". ASME - Mars 76.

[2] BoLLETER. - " Using transfer function measurements to determine energy propagation in fluid lines, with application to centrifugal pump systems ". CETIM 1981 - Congrès Intensimétrie Acoustique.
[3] Badie-Cassagnet, Bockhoff, Lambert. - Application de l'intensimétrie acoustique à l'identification des sources de pulsation de pression dans les circuits ". CETIM 1981 Congrès Intensimétrie Acoustique.

[4] LATOMBE, TAS. - "Détection multiple par les valeurs propres de la matrice interspectrale ". Traitement du Signal Vol. 3, 1986.

\footnotetext{
Adresses des auteurs

Monsieur D. Garreau$$
\text { EDF-DER }
$$

6, quai Watter

78400 Chatou

Monsieur Leducq

Société Bertin

BP 3

78370 Plaisir

Monsieur J.L. Trolle

EDF-DER

25, allée Privée

93206 Saint-Denis

Tél. : 48.29.90.00
} 
M. le Président. - Merci Messieurs. Je vous propose de répondre aux questions de l'auditoire.

M. DEsmet. - Avez-vous utilisé les techniques présentées pour l'étude des pompes de dimensions variées?

M. TRolle. - Oui, nous avons utilisé fréquemment ces techniques que ce soit sur des pompes de petite taille, genre pompes d'arrosage, ou que ce soit sur des pompes de taille plus importante comme les pompes alimentaires de centrale nucléaire que nous avons testées sur des boucles d'essais.

M. DESMET. - N'avez-vous pas rencontré des difficultés pour ces études de pompes, liées en particulier à des spectres de raies?

M. TROLLE. - Nous n'avons pas rencontré de difficulté particulière; le spectre de raies s'analyse de la même manière.

Dans certains cas, des problèmes sont apparus, mais ils étaient liés au fait que la pompe n'excitait pas uniquement le fluide mais aussi la structure. Cela se détecte soit par une chute de cohérence, soit par la présence d'une partie imaginaire non nulle sur la demi-somme des fonctions de transfert entre les capteurs extrêmes et le capteur médian. Actuellement, nous développons des techniques afin de caractériser ces propagations d'ondes dans la structure.

M. DESMET. - Dans un circuit peu bruyant, une pompe ne fournit une excitation convenable que sur quelques raies et nous avons rencontré des difficultés lors de la mesure de la célérité.

M. TROLLE. - Il est intéressant d'avoir un circuit peu réflecteur et assez bruyant. Notamment dans le cas du fonctionnement d'une pompe à son régime nominal, le spectre ne contient quasiment que des raies si le circuit est très peu bruyant; il risque alors d'être délicat d'estimer la célérité du son par la méthode des fonctions de transfert. Dans les fonctionnements à débit partiel, l'excitation associée à la pompe contient un spectre basse fréquence qui élimine ce problème.

M. PRENat. - Pensez-vous qu'on puisse appliquer une méthode d'intensimétrie acoustique dans un circuit d'essai de turbine hydraulique caractérisé par:

- une conduite divergente à l'aval finissant dans un niveau libre,

- des fonctionnements en cavitation notamment avec présence de torches?

M. TROLLE. - Premier problème: Le modèle utilisé n'est valable que pour une tuyauterie droite. Si l'on voulait faire la même analyse pour une conduite divergente, il faudrait dévelop. per un modèle adapté. Cela peut se mettre en équations, il suffit donc d'adapter les techniques utilisées.

Deuxième problème: En ce qui concerne la cavitation, il faudrait évaluer une célérité du son en fonction de la distance dans l'axe de la conduite. C'est réalisable en augmentant le nombre de capteurs, et en lissant les différentes célérités obtenues afin de dégager une loi d'évolution.

Troisième problème: Il est nécessaire que le modèle d'ondes longitudinales planes soit toujours valable; c'est-à-dire que le rapport entre le produit de la fréquence du phénomène acoustique par le diamètre et la célérité reste inférieur à $1(f D / c \ll 1)$.

M. THIRRIOT. - Pourriez-vous donner un complément d'informations à propos de l'effet de la cavitation sur la vitesse du son dans la configuration étudiée et sur un plan plus général ?

M. TROLLE. - Dans la configuration étudiée, nous avons constaté que la cavitation se retrouvait loin de la vanne. Différentes mesures par intensimétrie ont été réalisées le long de la tuyauterie à l'aval de la vanne; les résultats ont montré que la célérité était de $380 \mathrm{~m} / \mathrm{s}$ à $50 \mathrm{~cm}$ de la vanne, de $420 \mathrm{~m} / \mathrm{s}$ à $80 \mathrm{~cm}$ et de $470 \mathrm{~m} / \mathrm{s}$ à $4,75 \mathrm{~m}$. Cependant, à l'amont de la vanne, la valeur de la vitesse du son était de $1260 \mathrm{~m} / \mathrm{s}$ ce qui prouve que la cavitation s'était certainement résorbée lors du passage dans la pompe.

M. THIRRIOT. - Vous avez pris en compte la variation de la célérité. Pourriez-vous en dire un mot de plus ?

M. TROLLE. - Les capteurs étaient positionnés de manière équidistante à l'aval de la vanne ce qui a permis de déterminer la vitesse du son par la méthode des fonctions de transfert. Toutes les demi-sommes de fonctions de transfert obtenues possédaient une allure de fonction cosinus. Cela s'explique par le fait que la cavitation émet certes dans toutes les directions, mais que seule la partie émise dans l'axe de la tuyauterie est susceptible de se propager en ondes planes. Ainsi, la partie qui se propage dans l'axe de la tuyauterie possède une relation de phase entre 2 mesures successives imposées par la propagation, tandis que la partie émise restante fournit une relation de phase aléatoire en ces 2 mêmes mesures de telle sorte que si l'on moyenne suffisamment les signaux, les interspectres notamment, il ne subsiste que la partie liée aux fluctuations propagatives. A l'inverse, les autospectres de pression contiennent l'ensemble des fluctuations et permettent de ce fait d'estimer les fluctuations à caractère non propagatif, ce que nous appelons les fluctuations locales. 